\title{
Research on Key Technology of New Concept Tyre Building Production Line
}

\author{
Menglong Cao \\ Dept. of Automation and Electronic Engineering, Qingdao University of Science \& Technology, Qingdao, China \\ cml_qd@tom.com
}

Guangbo Wang, Lina Sun

Dept. of Automation and Electronic Engineering, Qingdao University of Science \& Technology, Qingdao, China cml_qd@tom.com

\begin{abstract}
Tyre building production line gradually transits from stand-alone production to combined production mode. The transformation of work mode from traditional serial intermittent to the parallel continuous has been the key technology research of tire production enterprise. And intelligent robots and other automated equipment have been the first choice of the tyre enterprise's production line. Considering the combination of the equip ment between upper and lower processes in tyre production line, the manual operations in some processes replaced by intelligent robots will improve production efficiency of tyre production enterprise, and will make outstanding contribution in reduce process losses and reduce production costs. This article studies on the key technology of combined application in production line, and makes simulation comparison for the same technical process that using different scheme, to prove the priorities and superiorities of combined production line relatives to the traditional production mode.
\end{abstract}

Index Terms-Tyre Building System, Combined Production Line, Process Simulation

\section{Introduction}

New requirements of equipment quality, efficiency and costs are put forward by Market competition of tyre building machine. To break the structure design concept of two drum machine, three drum machine and four drum machine from technological form and found new structure of tyre build ing machine is the new topic that the tyre manufacture industries have to face. Mechanization, linkage and automation of tyre building machine are developing quickly. At present, the building equipment consists of perfect building drums, belt building drums, functional feed servers, transfer rings, pressure roller devices and automatic laser indicator lights. In build ing process, all kinds of glue stock array, and rubber parts' let-off, fixed- length, cutting off, end delivery, splice, joint, traveler's deliver and location are all executed mechanization and linkage. All these movements deliver signal to PLC by kinds of sensing elements, controlled by computer to realize a measure of automation production process. Now, each drum of building machine bears so much duty that many processes have to be finished on this drum will certainly cause feeder complicated and mutual interference of linkage action that bad for automation control of building process [1]. So, feeder structure can be decomposed if each process be finished by one drum, and great convenience will be brought for design of automation technology.

In industrialized countries, a lot of human, material and financial resources had invested to the research about technology and equipment of automatic tyre building for many years [2], but they are strictly confidential for those technologies and building equipment with production conditions. Analyzing on the efficiency index of automatic technology from some papers about C3M of Michelin(Command + Control+ Communication \& Manufacture),MIRS of Pirelli (Modular Integrated Robotized System), CCC of three seas (Continuous Cold Compounding), IMP of Goodyear(Integrated Manufacturing Precision Assembly Cellular Technology), MMP of (Modular Manufacturing Process), ACTAS of Bridgestone (Automatic continuous tyre building system) [3,4], we can get that their new technology have below in common: automatic operation launched comprehensively centering on building; using low temperature continuous mixing rubber process; removal and merger process of components' prefabricate and park. But, C3M of Michelin and MIRS of Pirelli seemingly more epoch-making significance relatively because they almost involve the whole production process: from product designing, sample making model designing, materials procurement, milling, calendaring, extrusion, building, curing to final product storage. Automatic tyre building technology is the most promising and active future trend [5]. 


\section{Combined Building System Theory and Simulation Modeling}

According to the point of complex system in the view of system theory, we can model combined building system and simulate the key technology of combined building system based on the model. Combined production line is a complicate system in which input is material, equipment, tool, energy, human, manufacture theory, process and information relates to manufacturing and output is a qualified product [6]. In the process of model the reconfiguration of system should also be considered, that is to say, internal cellular construction of system can be readjusted and all information of sensors can be fused without changing the function of system when we design high-performance tyre building system. The direct objective of reconfiguration is to improve the internal structure of system to reflect changes in demand, to better meet clients' requirement and maximum service life. When a fault occurs, production line include characteristic design of reconfiguration line could switch or replace the fault unit by means of reconfiguration internal system which plays a complementary and redundancy role. After a fault occurs, because of its' automatic element, combined production line could take the appropriate measure caused the system from fault state to normal working state by means of fault detection and location. Fig. 1 and Fig. 2 display the continuous work flow diagram of combined reconfigurable building system and internal dynamic.

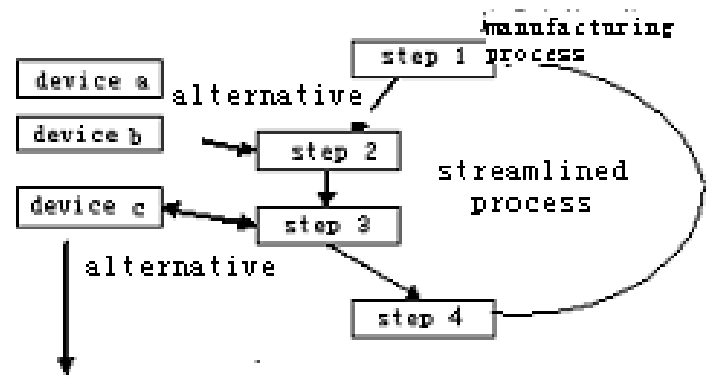

Fig.1. Continuous work flow diagram of combined reconfigurable tyre building system.

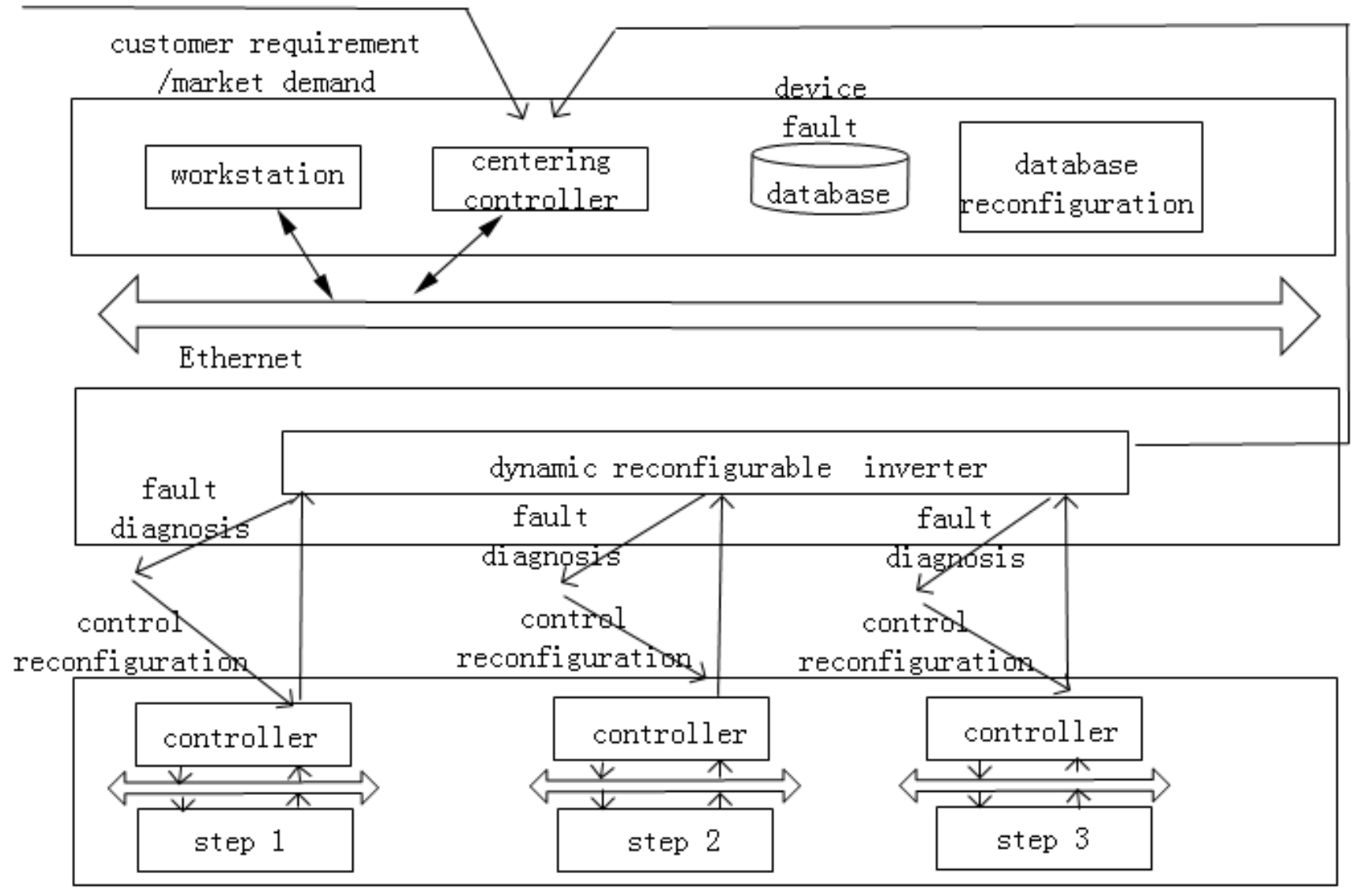

Fig.2. Dynamic reconfigurable schematic diagram of combined tyre building system

Fig. 3 shows the decomposition for each step in building process based on the research above, then to establish the model of combined production line according to the decomposed step.

Using computer to simulate production process need to build virtual manufacturing environment and it's corresponding virtual manufacturing device [7]. Each virtual device is equivalent to a component that should more completely reflect characteristics of physical device, such as geometrical features, material characteristics, motion information and so on. So, digitize the system model according to it's characteristics to establish simulation model of virtual device. Virtual device model is a mapping of physical 
device function and form feature in virtual environ ment. Because the functional specificity of physical device decide the geometrical features of virtual device model, from the two aspects-geometrical model and motion control to analyze the function feature and geometric feature in building virtual device model, and divide the virtual device model to geometrical model and motion control model.

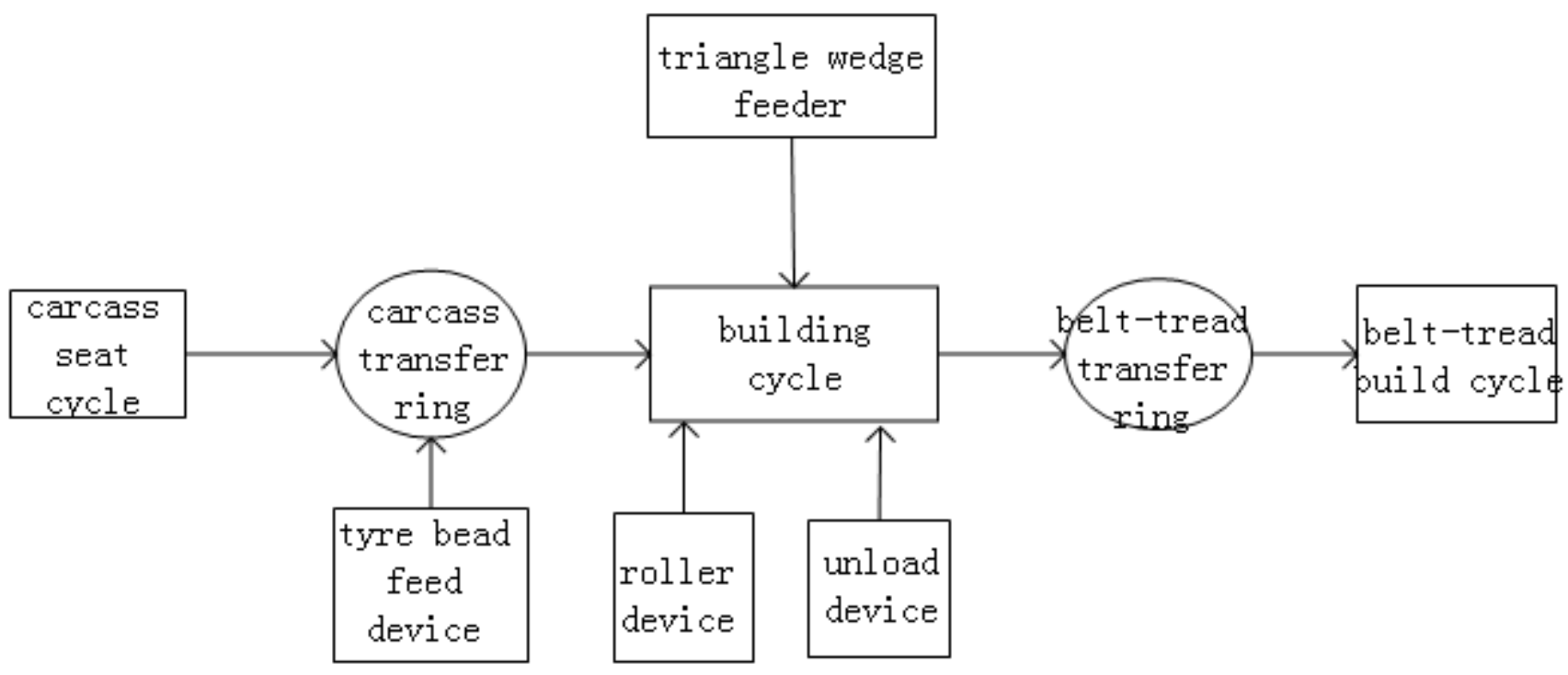

Fig.3. Process decomposition of tyre building system

Geometric model and description of form feature of physical device expressed the basic shape information of physical equipment, such as form of building drums, feeding servers, loading and unload devices. These geometric models will do some corresponding motions according to the input control data external, under the control of motion control model, and these motions could express the active of physical device, for instance, feed of feeding frame, rotation of main spindle, action of robot and motion of moving drum. All kinds of control function indicated by motion control model, which could output corresponding control information undertake handling, judg ment depends on external input control information, to drive position and motion state of relative geometric model to change, realize virtualization of behavior of physical model device.

\section{Units and Function of Combined Building System}

\section{A. System Units}

Moving drum is a transfer-device with installation rail, also some feeding devices can be disposed around the tyre building production line, such as belt servers, cap ply servers, tread servers, carcass servers, pressing devices. All those devices and servers will make a perfect combined production line of tyre building. Reducing manual operation content is the first purpose of system design, not only for increasing production capacity, but also improving the reconfiguration. Combined tyre building production line is highly automatic that content of manual operation is reduced to a minimum and it's high productive capacity can be seen.
Belt automatic server is designed to unit with automatic measurement, cutting control, exact orientation function, include: (1) mainframe connector, with two transfer-devices drive rolling race, and the top of each device have a belt cutting system. (2) two let-off stations, use standard scroll or make-to-order. (3) belt cutting system. (4) belt orientation-centering system, to realize exact docking.

Cap ply server include: (1) let-off, for cap ply's operation with flexible width (2) loop control (3) sticking device(4) cap ply cutter (5) scroll.

Carcass server, that placed in front of belt-carcass B\&T drum easy to lay up tread rubber, contains mainframe with rolling race and orientation device will oriented by groove in center or edge of tread rubber. Carcass servicer applied to tread rubber with flexible width, and height of carcass orientation device is adjustable.

Control unit uses programming logic controller in industry, adopt servo-drive to realize exact synchronous to store fault diagnosis, tyre specification and maintenance guideline in upper computer.

\section{B. Unit Function of System}

\section{1) Automatic Tyre Loading and Unload Process}

To combine robot with mainframe will make production line more flexible, function and processing efficiency are further optimized. Carcass placed on the upper conveying chain is transferred to the direction of building drum one by one, then robot takes the first carcass to the position of holding device in front of building machine and transferred to the building drum by holding device. Green tires will be unloaded by 
robot and transferred to the lower conveying chain after a small processing table to be sent out. It is an automatic process of loading and unloading. Application of robot tyre operation module can improve operation efficiency of tyre bead. Design scheme revolves from easy loading and unloading system to the robot storage and recognition system to realize load tyre bead and remove raw tyre automatically through analogue simulation, which can improve system stability and working efficiency. Fig. 4 shows diagram that robot operate the device.
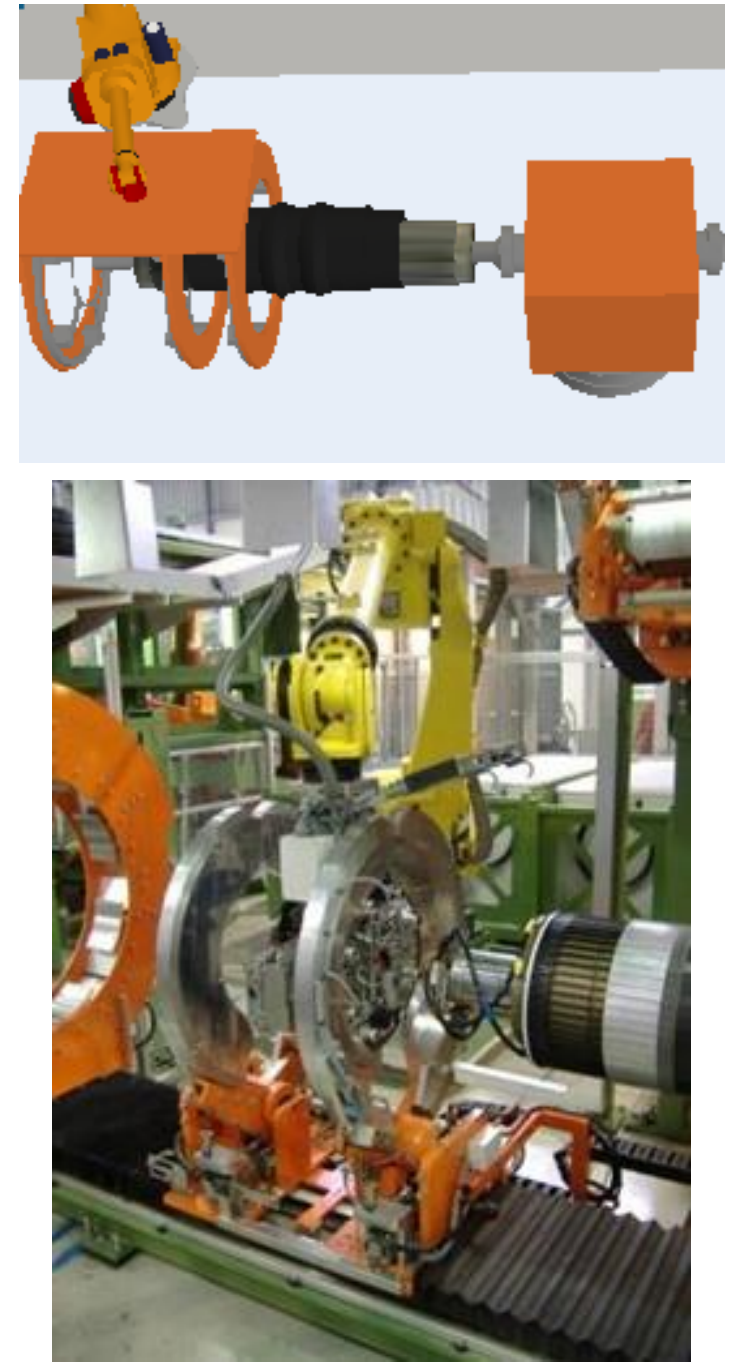

Fig.4. Handling tyre automatically with robot

The device is very useful to improve labor productivity and alleviate worker labor strength undoubtedly. Though floor area of combined production line constructed may increase, analyzed on capacity the adverted effects caused by it can be ignored.

\section{2) Automatic Belt Feeding Process}

Automatic belt server can achieve belt cutting control, deliver joint to the B\&T drum, belt end-to-end automatically. Now, there are some difficulties to realize complete automation for domestic belt server in the current domestic conditions. But, improve mechanization and automation degree of building process, expel product defects caused by personal factors is the important way to improve building quality of radial tyre, and is one of the subject that radial tyre building machine must solve.

Automatic belt server can be a module of combined building system. Design of combined belt server makes manufacture of tyre building more flexible, using designed module can make the change time almost negligible when another angle or width of belt feeding is need which improve the working efficiency. Fig.5 is the diagram of belt automatic feeding module.
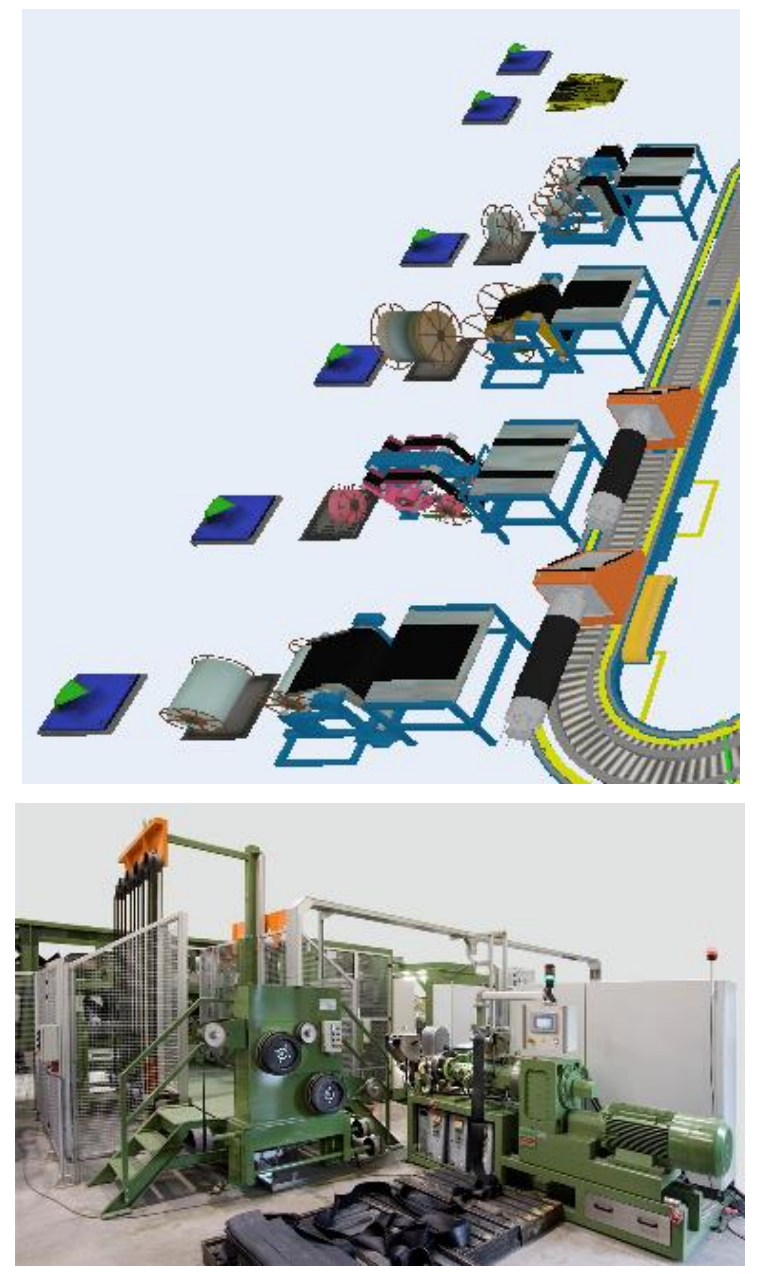

Fig.5. Diagram of combined belt automatic feeding system

\section{3) Auxiliary Processes}

Step1 Carcass seat process. Breaking process of carcass seat into multi station implement, vehicle-mounted B\&T drum and driving box operate synchronistically located each station to complete carcass seat, and disposed a number of mobile robot to form a work mode that multi station carcass component seat in parallel.

Step2 Belt seat process. Breaking process of belt seat into multi station implement, rotary belt-carcass B\&T drum and driving box operate synchronistically located each station to complete seat, form a work mode that multi station belt-carcass component seat in parallel. 
Step3 Building roller process. Breaking process of building roller into multi station implement, vehicle-mounted(or rotary) building drum and driving box operate synchronistically located each station to complete building roller, and disposed a number of mobile robot to form a work mode that multi station tyre building roller.

Step4 Carcass transfer and belt-carcass transfer process. Carcass transfer ring and belt-carcass transfer ring adopt upper steering mechanism.

According to the requirement of building process, combined tyre build ing system's structure is simple and installation and adjustment easily, formed characteristic distribute fault tolerant control, fast driving and exact location, auxiliary equipment of main drum can feed materials synchronistically when the main drum is turning.

Changing the traditional intermittent serial work mode, achieve continuous parallel work mode, improving the system stability and reliability in operation, the human-computer interface may call and carry out various production process, display, record production status, can do remote debugging, monitor, etc. It has incomparable superior over trad itional type in building precision, process parameter setting and stability.

\section{Simulation of Combined Production Line Based on Flexsim}

Flexs im is a kind of commercial simulation software for discrete event produced by America Flexsim Software Production. Flexsim uses object-oriented technology, and has three dimensional display functions. The software provides the original data fitting, input modeling, graphical model building, virtual reality shows, operate model to do simulation experiment, optimize the results and the formation of 3D animation image files and other functions, also provides interface with other tool software. The ma in advantage of Flexsim is embodied in modeling quick and simple and strong simulation analysis ability.

Flexs im simulation modeling has the following five basic steps:

Step1 Setting layout. According to the designed physical system before modeling, drag the object from "object library" to the simulation view window in proper position.

Step2 Define the "flow". Connect corresponding port, constructing logic flow of simulation model according to logical relationship of objects to

Step3 Set parameters. According to the physical system characteristic described to set object parameters.

Step4 Running model. Compile the model, then reset and run this model.

Step5 Analysis of simulation results. Evaluate system schemes according to the simulation results to, and modify parameters according to the simulation target to form the different system scheme. Comparing each scheme to achieve optimize purposes.

According to the model above and simu lation steps to establish original process and simulation environment of improved process proved the feasibility and superiority improved scheme. Fig.6 shows the simulation comparison model.

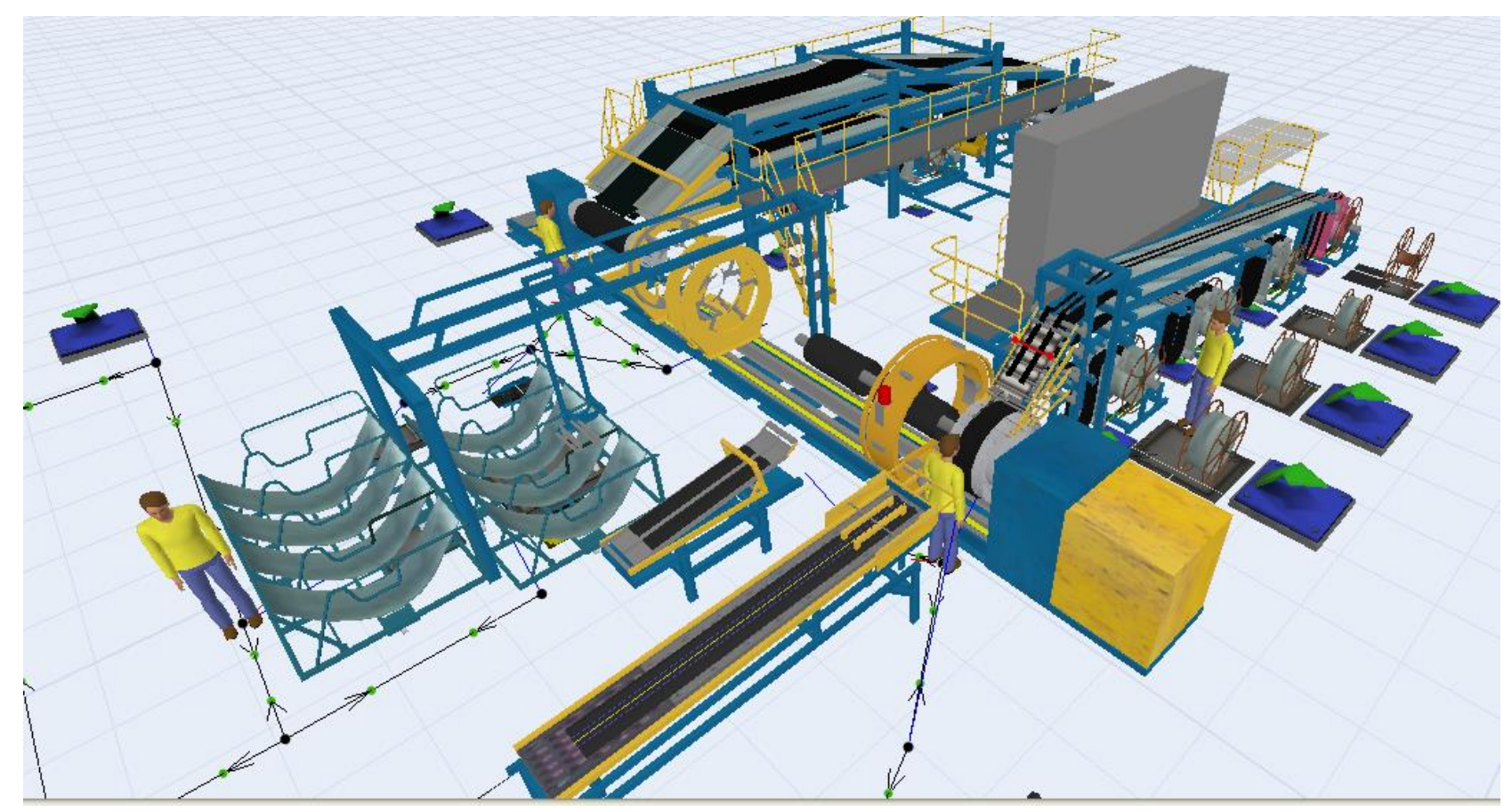

Fig.6. Simulation comparison model (a) 


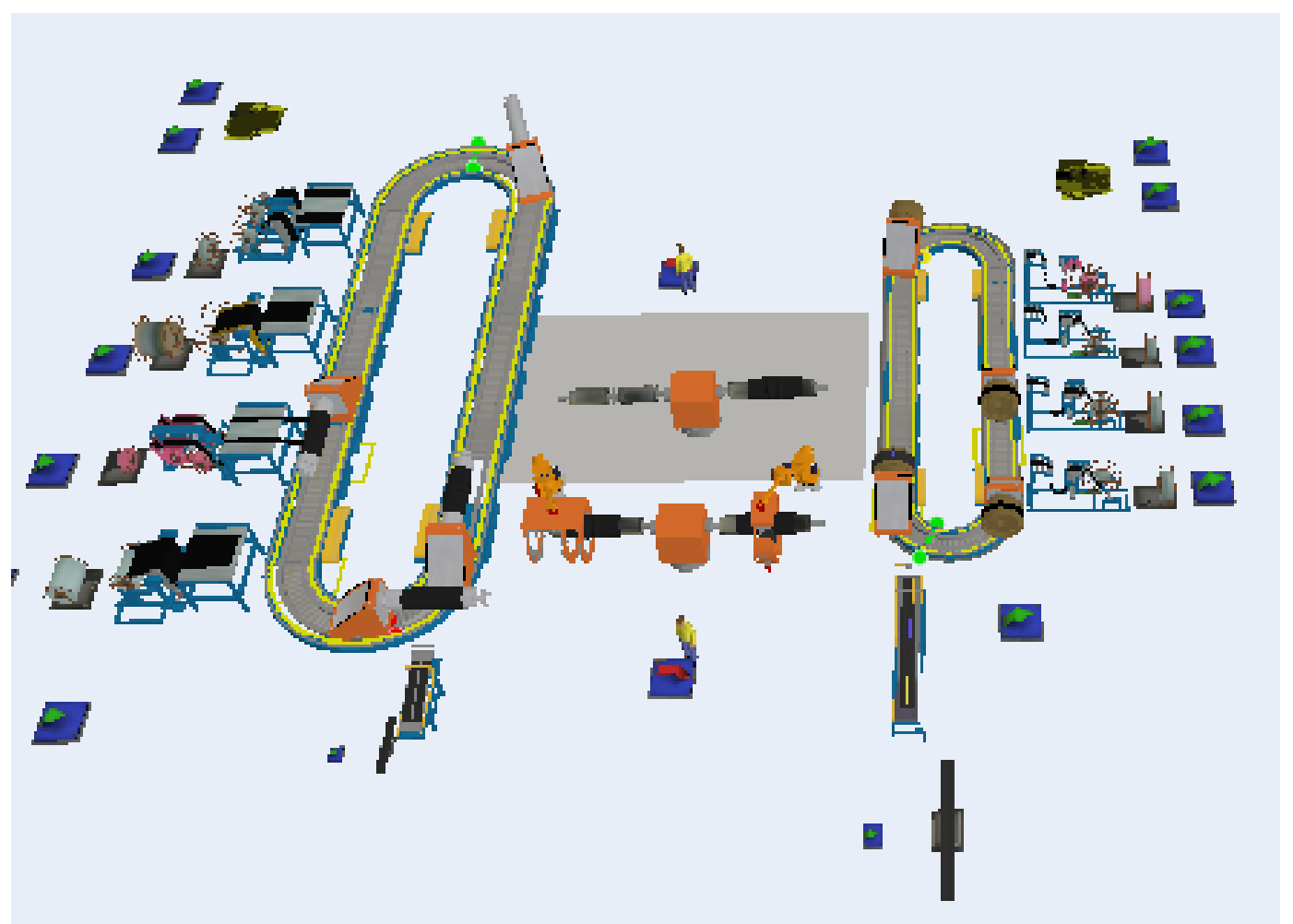

Fig.6. Simulation comparison model (b)

Using computer simulation, visualization, motion modeling and motion simulation, data decoding technology, we have formed the running process of combined tyre building production line with visual mode. Based on it, user can evaluate adopted equipment, layout, etc, and make capacity analysis and comparis on to obtain high performance product.

\section{Conclusion}

In premise of application, digest and absorb the key technology of foreign advanced manufacturing system, pay attention to human-computer interface's optimization in design scheme with no overboard automation, establish comparative perfect information system as far as possible, fully develop benefit of computer management, let advanced automation equipment and ordinary equipment coexist, allow manual intervention in some processes, forming applicability automation technology solutions. The reference of foreign experience of mechanical manufacturing low cost automation technology is a technical route accord with national condition. Our tyre manufacture enterprise have plenty of universal equipment, when develop modern mechanical automation technology, rationally adjust the original equipment layout to add some intelligent device with original equipment primarily, full display the advantage of computer automatic management and man's creativity to construct the autonomous unitization production system that people-centered and information automatic as the pilot, it will provide a new way for our automation technology development of tyre industry with less investment, rapid, high efficiency and suitable for our national condition.

\section{References}

[1] Xue Peijun. The discussion on development trend of machine manufacturing $[\mathrm{J}]$.An yang engineering college academic journal,04 (2005)14-16.

[2] Weihong Xie, Xiaochao Cheng, Luan Jiang. An Empirical Research of Relationship between Corporate Strategy [J].Manufacturing Flexibility and Performance,(2009)12-14.

[3] D. Shin, R. A. Wysk and L. Rothrock. A formal control-theoretic model of a human-automation interactive manufacturing system control [J]. International Journal of Production Research, 44(2006)4273-4295.

[4] Z. M. Bi, S. Y. T. Lang, W. Shen and L. Wang. Reconfigurable manufacturing systems: The state of the art $[\mathrm{J}]$. International Journal of Production Research, 46(2008)967-992.

[5] Xue Jiabing,E Mingchen. The exploitation of FMS plant control system based on flexsim[J]. Chinese manufacturing informatization, 1 (2007)38 - 41.

[6] A. M. Law.How to build valid and credible simulation models[C].In Proceedings of the 2005 Winter Simulation Conference, (2005)24-32.

[7] B.Y. Ekren and A. M. Ornek. A simulation based experimental design to analyze factors affecting 
production flow time[J].Simulation Modeling Practice and Theory,16(2008)278-293

[8] M. Savsar and M. Aldaihani. Modeling of machine failures in a flexible manufacturing cell with two machines served by a robot[J].Reliability Engineering \& System Safety, 93(2008)1551 1562.

[9] Zhang Weide, Yan Hongsen, Xu Cheng. The simulation and application of production line based on Flexsim [J]. Industrial control computer,9 (2005) 46-47.

CAO Meng-long $(1971-)$, male, Qingdao, China, Associate Professor, Ph.D., his research directions include autonomous navigation, multi-sensor information fusion and intelligent control.

How to cite this paper: Menglong Cao,Guangbo Wang, Lina Sun,"Research on Key Technology of New Concept Tyre Building Production Line", International Journal of Intelligent Systems and Applications(IJISA), vol.4, no.8, pp.46-52, 2012. DOI: $10.5815 /$ ijisa.2012.08.06 